\title{
The Future of Psychotherapy: Should Cognitive- Behavioral Therapy be Utilized Instead of Psychodynamic Therapy?
}

\author{
Elaine A Burke* \\ Department of Psychology, USA
}

ISSN: 2639-0612

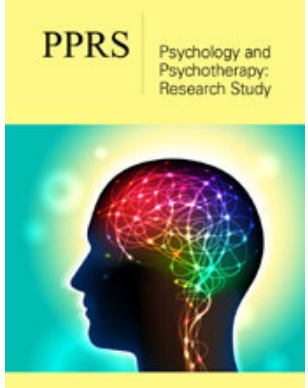

*Corresponding author: Elaine A Burke, Department of Psychology, USA

Submission: 泟 October 23, 2019

Published: 眥 October 29, 2019

Volume 2 - Issue 5

How to cite this article: Elaine A Burke. The Future of Psychotherapy: Should Cognitive-Behavioral Therapy be Utilized Instead of Psychodynamic Therapy? Psychol Psychother Res Stud. 2(5). PPRS.000549.2019.

DOI: 10.31031/PPRS.2019.02.000549

Copyright@ Elaine A Burke, This article is distributed under the terms of the Creative Commons Attribution 4.0 International License, which permits unrestricted use and redistribution provided that the original author and source are credited.

\section{Opinion}

There has been an evolution of therapeutic approaches in psychotherapy since its inception. Sigmund Freud is often credited with the initial development of psychotherapy using a psychodynamic approach (late 1800s). The emphasis of this approach was to bring repressed or unconscious memories into conscious awareness so that they can be explored within the context of the therapeutic relationship and the resulting transference. Devotees of this theory thought that long term therapy was needed to repair the damage due to early childhood experience. The most effective form was considered to be intensive, long-term treatment for an in-depth exploration of the person's past experience. Around the same time as the development of psychodynamic therapy, a behavioral approach was introduced. At its simplest form, a stimulus was associated with a response. Pavlov was associated with placing food in front of his dogs and their consistent response was to salivate. However, researchers argued that for people, their reactions may not be so automatic and predictable, but instead determined by learned behavior. In addition, people have thoughts or belief systems that influence their responses. Therefore, if the intermediary thought process (belief system) was identified and transformed, more adaptive behavior could occur. While other forms of therapy have been developed and utilized, cognitive behavior and psychodynamic treatment have tended to be the most commonly used.

During the twentieth century, psychology training programs taught one or more of these forms of intervention. While some programs focused on either cognitive behavioral or psychodynamic approaches, most training institutions provided instruction in more than one theoretical orientation. Psychologists identified with primarily one of these theoretical conceptualizations or a more eclectic or integrated theoretical perspective. Clients seeking services from agencies or individual practitioners could generally find services in either one of these modalities. Psychodynamic approaches were often considered to be more beneficial for in-depth treatment for more complex problems, while cognitive behavioral approaches might be used with depression, anxiety, fears or specific problems such as weight loss or smoking cessation when specific behaviors could be changed to correct the problem. These treatments were generally covered by insurance. However, during the late twentieth century, insurance companies began to incorporate HMOs (health maintenance organizations) to evaluate the need for treatment and oversee the treatment process in order to reduce and control the high cost of care. Based upon the diagnosis and anticipated progress, a certain number of sessions were authorized. If people did not meet the criteria for an extension of services, then sessions were no longer covered by that insurance carrier. Due to the desire for rapid change in order for the treatment to be covered, shorter treatments became more common (generally cognitive behavior therapy). Behavioral approaches conformed to the guidelines of establishing identified goals, specific treatments, and observable/measurable change. These treatments (again, generally cognitive behavioral) were described as evidence-based treatments. Over time, additional evidence-based treatments (based upon a cognitive-behavioral perspective) such as TF-CBT and DBT were developed. Manualized, packaged approaches could also be provided by clinicians with less training who were not psychologists (who were paid less) thus further reducing the cost of treatment. The direction of psychotherapy into more cognitive behavioral evidence-based practices raises some 
important questions. Are these cognitive behavioral approaches more effective than psychodynamic treatment?

Are they effective for all clients, and if not, does the field of psychology continue to promote psychodynamic treatment? Psychologists know when cognitive behavioral approaches are not successful, but the question is whether they have been adequately trained to implement alternative approaches that may be more effective? There has been some research comparing the effectiveness of cognitive behavioral and psychodynamic treatment. Dreissen [1] found minimal differences between the two approaches for the treatment of depression and suggested that knowledge of client factors would influence the best course of treatment. Goldstein [2] found similar results, and thought that the severity, etiology, and length of treatment affected outcome when treating depression. Dreissen [3] determined that psychodynamic treatment can be as beneficial as CBT if patient factors other than the reduction of depressive symptoms was considered. Generally, the studies indicated that the effectiveness of both approaches is similar, and that client factors need to be considered in order to determine which treatment would provide better outcomes. Some research even favors the use of psychodynamic interventions.

Mulay [4] thought that psychodynamic approaches should be used in prisons (rather than the short-term behavioral approaches that are often used) as incarcerated individuals tend to have more complex issues, they are in prison for a long time, and there are treatment issues that are specific to being in a correctional setting. If the field of psychology uses primarily cognitive behavioral approaches, it is possible that a significant percentage of people who would receive the most benefit from a psychodynamic approach would not receive it. If this situation is true, then perhaps the overall mental health of the population may decrease. There could be some indicators of this phenomenon such as increased unemployment or disability due to a mental health disorder, poorer performance in academic settings, more suicides and perhaps more crimes. However, would this lack of appropriate treatment be recognized when there are so many societal factors that contribute to these problems? There may be some clients who improve somewhat with cognitive-behavioral evidence-based practices, but whose treatment could be much more successful with psychodynamic approaches. Are they being denied the highest quality of life due to receiving inadequate treatment for their needs? While it is important to evaluate treatment effectiveness, group studies of treatment efficacy cannot determine the best approach for vastly different clients. The field of psychology needs to continue to advocate for therapy utilizing a multitude of interventions so that individual clients can receive the form of treatment that is the most effective for them. Hopefully the future of psychotherapy will include a number of types of interventions, including psychodynamic. that are tailored to clients' needs in order to maximize their success in therapy and subsequent ability to handle life's challenges.

\section{References}

1. Driessen E, Smits N, Cuijpers P, Dekker JJM, Peen J, et al. (2016) Differential efficacy of cognitive behavioral therapy and psychodynamic therapy for major depression: A study of prescriptive factors. Psychol Med 46(4): 731-744.

2. Goldstone D (2017) Cognitive-behavioural therapy versus psychodynamic psychotherapy for the treatment of depression: A critical review of evidence and current issues. South African Journal of Psychology 47(1): 84-96.

3. Driessen E, Don FJ, Cuijpers P, Van HL, Peen J, et al. (2017) Cognitivebehavioral versus psychodynamic therapy for major depression: Secondary outcomes of a randomized clinical trial. J Consult Clin Psychol 85(7): 653-663.

4. Mulay AL, Kelly E, Cain NM (2017) Psychodynamic treatment of the criminal offender: making the case for longer-term treatment in a longer-term setting. Psychodynamic Psychiatry 45(2): 143-173. 